\title{
Simulation of non-isothermal free turbulent gas jets in the process of energy exchange
}

\author{
Muzaffar Hamdamov ${ }^{1 *}$, Akmal Mirzoyev ${ }^{1}$, Eshmurod Buriev ${ }^{1}$, and Nosirbek Tashpulatov ${ }^{2}$ \\ ${ }^{1}$ Institute of Mechanics and Seismic Resistance M.T. Urazbaeva Academy of Sciences of the \\ Republic of $U$ \\ ${ }^{2}$ Fergana Polytechnic Institute, Fergana, Uzbekistan
}

\begin{abstract}
This article proposes a numerical method for solving the propagation and combustion of a jet of a gas mixture in an axisymmetric satellite air stream. To model the process, the dimensionless equations of the turbulent boundary layer of reacting gases in the Mises coordinates are used. A two-layer four-point nonlinear boundary separation scheme was used to solve the problem in the Mises coordinates, and a second-order along the longitudinal coordinate was given. The iterative process was used because of the nonlinearity of the storage and displacement equations of substations. Individual results of the numerical experiment are presented.
\end{abstract}

\section{Introduction}

The development of methods for studying jet flows of multicomponent gases can be monitored in [1-7]. The dominance of convection over diffusion, the alternating coefficient for the convective term, makes it possible for local subdomains to appear in the computational domain with a large boundary of the desired function of the boundary and internal transition layers [8]. The presence of sources and drains in chemically reacting flows introduces additional difficulties into the picture of the process. Ultimately, this leads to serious difficulties in the numerical integration of equations of mathematical models.

The dominance of convection over diffusion, the alternating coefficient for the convective term, leads to the appearance in the computational domain of local subdomains with a large boundary of the desired function of the boundary and internal transition layers. The presence of sources and sinks in chemically reacting flows introduces additional difficulties into the picture of the process. Ultimately, this leads to serious difficulties in the numerical integration of equations of mathematical models. These difficulties in modeling and numerical solution of heat and mass transfer problems are the different scale of diffusion processes, convection, and source drains.

\footnotetext{
*Corresponding author: kamina.0691@,mail.ru
} 
In general, a strict quantitative calculation of the diffusion torch of the final size is extremely difficult. However, this task reflects the process in the main section of the jet, which is of the greatest practical interest, and it also has the greatest computational difficulties. On the one hand, the transport and conservation equations contain third-order terms concerning unknowns. On the other hand, unknown boundaries appear in the problem - the flame front and the boundary layer boundary.

Separate entry of gases into the zone of intense heat and mass transfer causes a tangential discontinuity in flow rates at the inlet section. This and the presence of a flame front lead to establishing a turbulent flow regime in a jet stream [1]. Therefore, the kinematic viscosity coefficient in the framework of the work was taken for a turbulent flow and a new modification of the L. Prandtl hypothesis was used $\varepsilon(x)=\chi b(x)\left|u_{\max }-u_{\min }\right|_{x}$, where $b(x)$ is cross-section jet $x$, calculated from the axis of the jet; $u_{\max }, u_{\min }$ are the largest and smallest value of the longitudinal component of the velocity vector in section $\mathrm{x}$.

A jet of flammable gas is considered, which flows out of a round nozzle with a diameter of $2 a$ and propagates in a satellite stream (warm space) of the oxidizer during diffusion combustion. Note that it is customary to distinguish between two forms of a direct jet torch - a flooded and a satellite torch. In the first case, it is about the expiration of a jet of fuel in the space flooded by a stationary oxidizing agent (for example, air), in the second - about the expiration of a stream of fuel in a parallel spiral oxidizer stream. Obviously, the second case is a general one and contains as a special case, if the velocity of the satellite stream is equal to zero, the problem of a flooded torch.

We believe that the velocity distribution in the exit section of the nozzle and in the confluent stream and the initial (at $\mathrm{x}=0$ ) distribution of temperature and concentration of fuel and oxidizer will be considered given, uniform, uniform, and stepwise.

Assuming an infinitely high rate of the chemical reaction of combustion and the same rate of convective and diffusive (turbulent) transfer of components, the flame front can be represented as an axisymmetric surface, in its main part close to a cylindrical one, delimiting the calculation area into two zones.

One of them - the inner zone is filled with fuel and combustion products. Fuel diffuses to the front surface from the inside and an oxidizer from the outside. Combustion products diffuse from the flame front in both directions, into the inner and outer sides of the torch. At the front itself, the concentration of each of the reactants is zero, and the concentration of combustion products is maximum. At the same time, turbulent flows of reacting gases, fuel, and oxidizer flowing to the front and burning on it, as noted above, are in a stoichiometric ratio.

Concerning the coefficient of turbulent viscosity, we used the modified Prandtl model we proposed taking into account the heterogeneity and volume compressibility of the medium, which has the form: $\varepsilon(x)=k\left(\frac{\rho_{1}}{\rho}\right)^{\beta} b(x)\left|u_{\max }-u_{\min }\right|_{x}$, where $k, \beta$ are selectable permanent models; $b(x)$ is cross-section jet $x$, calculated from the axis of the jet; $u_{\max }, u_{\min }$ are the largest and smallest value of the longitudinal component of the velocity vector in the section $x[9,10]$. 
We consider a jet of a mixture with a combustible gas that flows out of a round nozzle with the diameter $2 a$ with speed $u_{2}$ and propagates in a confluent oxidant stream, the speed of which is $u_{1}$. Each of the separately introduced mixtures has its own composition and its own thermophysical characteristics. Gases mutually diffuse, and active gases react on a thin surface of the flame front. The combustion rate is considered large enough that the fuel does not penetrate into the air zone, and oxygen in the air cannot be in the zone of the combustible mixture. Accordingly, in the fuel zone, there are fuel, reaction products, and chemically passive gases; and in the air zone, there are oxidizing agents, reaction products, and chemically inert gases

It is required to develop a numerical method and a software product with which it is possible to study the jet stream for various compositions of the main and satellite flow, their aerodynamic and thermodynamic parameters. In this case, it is required to reduce the number of equations of transport and conservation of the gas mixture components.

In the approximation of the theory of turbulent boundary layer, the system of equations of turbulent motion of a multicomponent gas in the presence of chemical interaction between the components, with the equality of the unit Lewis number for components ( $\left.L e_{i}=1\right)$ ), can be written in the form [1]:

$$
\left\{\begin{array}{c}
\rho u \frac{\partial u}{\partial x}+\rho v \frac{\partial u}{\partial r}=-\frac{d p}{d x}+\frac{1}{r} \frac{\partial}{\partial r}\left(\rho v_{t} r \frac{\partial u}{\partial r}\right) \\
\frac{\partial(\rho u r)}{\partial x}+\frac{\partial(\rho v r)}{\partial r}=0 \\
\rho u \frac{\partial c_{k}}{\partial x}+\rho v \frac{\partial c_{k}}{\partial r}=\frac{1}{S c r} \frac{\partial}{\partial r}\left(\rho v_{t} r \frac{\partial c_{k}}{\partial r}\right)+\omega_{k}, \quad(k=1 . . N)
\end{array}\right.
$$

where $u, v$ are averaged longitudinal and transverse (radial) components of the velocity vector $\left(m s^{-1}\right) ; x, r$ are longitudinal and radial coordinates $(m) ; \rho, T$ is density $\left(\mathrm{kg} \mathrm{m}^{-3}\right)$ and absolute temperature $(K)$ gas mixture; $p$ is static pressure $(p a)$; $\operatorname{Pr}, S_{c}$ is turbulent analogues of Prandtl and Schmidt numbers; $c_{k}$ is mass concentration $k-$ is the gas component in the mixture $\left(\mathrm{kg} \mathrm{kg}^{-1}\right) ; \omega_{k}$ is mass formation/disappearance rate $k-$ is the gas component $\left(\mathrm{kg} \mathrm{m}^{-3} \mathrm{~s}^{-1}\right) ; v_{t}-$ is kinematic coefficient of turbulent viscosity or coefficient of turbulent exchange $\left(m^{2} s^{-1}\right)$.

The gas mixture is assumed to be perfect; therefore its state satisfies the MendeleevClapeyron equation $p=\rho R_{0} T / m$, where $m=\left(\sum_{k=1}^{N} m_{k} / c_{k}\right)^{-1}$ and $m_{k}$ is molar masses of 
the gas mixture and $k-$ is the gas component $\left(\mathrm{kg} \mathrm{mole}^{-1}\right) ; R_{0}$ is universal gas constant (8.341 Dj mole $\left.\mathrm{D}^{-1}\right)$.

The diffusion combustion model is generalized as applied to a complex composite combustible mixture, including several combustible components. The main essence of generalization is the formation of a united front for all combustible coefficients. Based on the model, the combustion of a mixture of methane and carbon dioxide in the air was studied. The features of the propagation of an axisymmetric jet of a combustible mixture in airspace were studied depending on the mass fraction of methane in the combustible mixture. In general, when simulating the combustion process, the main problem is to reduce the number of equations for the conservation and transfer of matter of $N$ components. Above, we analyzed the methods for introducing the Schwab - Zeldovich function and relatively excess concentration with the closure conditions for the equations for diffusion combustion models, the final rate of the chemical reaction, and chemical equilibrium.

The basic differential equations of a multicomponent turbulent boundary layer of reacting gases under the assumption that the turbulent Lewis number is equal to unity ( $\mathrm{Le}=$ 1) can be written as[11-13]

$$
\left\{\begin{array}{l}
\rho u \frac{\partial u}{\partial x}+\rho \vartheta \frac{\partial u}{\partial r}=\frac{1}{r} \frac{\partial}{\partial r}\left(\rho v_{t} r \frac{\partial u}{\partial r}\right)-\frac{\partial p}{\partial x} \\
\rho u \frac{\partial\left(c_{p} T\right)}{\partial x}+\rho \vartheta \frac{\partial\left(c_{p} T\right)}{\partial r}=\frac{1}{\operatorname{Pr}} \frac{1}{r} \frac{\partial}{\partial r}\left(\rho v_{t} r \frac{\partial\left(c_{p} T\right)}{\partial r}\right)+ \\
+u \frac{\partial p}{\partial x}+\rho v_{t}\left(\frac{\partial u}{\partial r}\right)^{2}-\sum_{i=1}^{N} h_{i}^{*} \omega_{i}
\end{array}\right.
$$

Multiplying the first equation by $u$, we get:

$$
\left\{\begin{array}{l}
\rho u \frac{\partial\left(u^{2} / 2\right)}{\partial x}+\rho \vartheta \frac{\partial\left(u^{2} / 2\right)}{\partial r}=\frac{u}{r} \frac{\partial}{\partial r}\left(\rho v_{t} r \frac{\partial u}{\partial r}\right)-u \frac{\partial p}{\partial x} \\
\rho u \frac{\partial\left(c p^{T)}\right.}{\partial x}+\rho \vartheta \frac{\partial\left(c_{p} T\right)}{\partial r}=\frac{1}{\operatorname{Pr}} \frac{1}{r} \frac{\partial}{\partial r}\left(\rho v_{t} r \frac{\partial\left(c p^{T)}\right.}{\partial r}\right)+ \\
+u \frac{\partial p}{\partial x}+\rho v_{t}\left(\frac{\partial u}{\partial r}\right)^{2}-\sum_{i=1}^{N} h_{i}^{*} \omega_{i}
\end{array}\right.
$$

By adding the corresponding thresholds of the system, we form the following equation: 


$$
\begin{gathered}
\rho u \frac{\partial\left(u^{2} / 2+c_{p} T\right)}{\partial x}+\rho \vartheta \frac{\partial\left(u^{2} / 2+c_{p} T\right)}{\partial r}=\frac{u}{r} \frac{\partial}{\partial r}\left(\rho v_{t} r \frac{\partial u}{\partial r}\right)+ \\
+\frac{1}{\operatorname{Pr} r} \frac{1}{r} \frac{\partial}{\partial r}\left(\rho v_{t} r \frac{\partial\left(c_{p} T\right)}{\partial r}\right)+\rho v_{t}\left(\frac{\partial u}{\partial r}\right)^{2}-\sum_{i=1}^{N} h_{i}^{*} \omega_{i} \\
\frac{u}{r} \frac{\partial}{\partial r}\left(\rho v_{t} r \frac{\partial u}{\partial r}\right)+\rho v_{t}\left(\frac{\partial u}{\partial r}\right)^{2}=\frac{1}{r} \frac{\partial}{\partial r}\left(\rho v_{t} r \frac{\partial\left(u^{2} / 2\right)}{\partial r}\right) .
\end{gathered}
$$

we simplify using this equality:

$$
\begin{gathered}
\rho u \frac{\partial\left(u^{2} / 2+c_{p} T\right)}{\partial x}+\rho \vartheta \frac{\partial\left(u^{2} / 2+c_{p} T\right)}{\partial r}=\frac{1}{r} \frac{\partial}{\partial r}\left(\rho v_{t} r \frac{\partial\left(u^{2} / 2\right)}{\partial r}\right)+ \\
+\frac{1}{\operatorname{Pr}} \frac{1}{r} \frac{\partial}{\partial r}\left(\rho v_{t} r \frac{\partial\left(c_{p} T\right)}{\partial r}\right)-\sum_{i=1}^{N} h_{i}^{*} \omega_{i} . \\
h=c_{p} T+\frac{u^{2}}{2} . \\
\rho u \frac{\partial h}{\partial x}+\rho \vartheta \frac{\partial h}{\partial r}=\frac{1}{r} \frac{\partial}{\partial r}\left(\rho v_{t} r \frac{\partial\left(u^{2} / 2\right)}{\partial r}\right)+\frac{1}{\operatorname{Pr}} \frac{1}{r} \frac{\partial}{\partial r}\left(\rho v_{t} r \frac{\partial\left(c_{p} T\right)}{\partial r}\right)-\sum_{i=1}^{N} h_{i}^{*} c_{i} .
\end{gathered}
$$

The total enthalpy of the gas mixture has the form

$$
\begin{aligned}
\rho u \frac{\partial H}{\partial x}+\rho \vartheta \frac{\partial H}{\partial r} & =\frac{1}{r} \frac{1}{\operatorname{Pr}} \frac{\partial}{\partial r}\left(\rho v_{t} r \frac{\partial}{\partial r}\left(H+(\operatorname{Pr}-1) \frac{u^{2}}{2}\right)\right) . \\
H & =c_{p} T+\frac{u^{2}}{2}+\sum_{i=1}^{N} h_{i}^{*} c_{i} .
\end{aligned}
$$

\section{Methods}

Enter discrete coordinates $\xi_{i}=i h_{\xi}$ and $\psi_{j}=j h_{\psi}$, as well as discrete functions $u_{i, j}, v_{i, j}, H_{i, j}, v_{t i}$. From the known values of the i-th layer, the values of $i$ - th layer.

The finite-difference representation of differential equations is feasible concerning the dimensionless equation of total enthalpy $[14,15]$ :

$$
\frac{\partial H}{\partial \xi}=\frac{1}{\psi} \frac{\partial}{\partial \psi}\left[\frac{\rho^{2} v_{t} u}{\psi}\left(2 \int_{0}^{\psi} \frac{\psi d \psi}{\rho u}\right) \frac{\partial}{\partial \psi}\left(H-f u^{2}\right)\right] .
$$


The condition of symmetry of the field of total enthalpy with respect to the $\mathrm{x}$-axis is realized as follows. We assume that $\psi=h$ small argument increment $\psi$ at $\psi=0$. Then the expression in parenthesis under the derivative on the left side of the equation is written as $2 \int_{0}^{\psi} \frac{\psi d \psi}{\rho u} \approx 2\left(\frac{0}{(\rho u)_{\psi=0}}+\frac{h}{(\rho u)_{\psi=h}}\right) \frac{h}{2}=\frac{\psi^{2}}{(\rho u)_{\psi=0}}$. Then the components of the right side of the equations can be represented as:

$$
\begin{aligned}
& \frac{1}{\psi} \frac{\partial}{\partial \psi}\left(\frac{\rho^{2} v_{t} u}{\psi} \frac{\psi^{2}}{(\rho u)_{\psi=0}} \frac{\partial}{\partial \psi}\right) \approx \frac{1}{\psi} \frac{\partial}{\partial \psi}\left(\rho v_{t} \psi \frac{\partial}{\partial \psi}\right)= \\
& =\frac{\partial \rho v_{t}}{\partial \psi} \frac{\partial}{\partial \psi}+\frac{1}{\psi} \rho v_{t} \frac{\partial}{\partial \psi}+\rho v_{t} \frac{\partial^{2}}{\partial \psi^{2}} \approx 2 \rho v_{t} \frac{\partial^{2}}{\partial \psi^{2}}
\end{aligned}
$$

Here the first term fell out, since $\frac{\partial}{\partial \psi}=0$. The second term was applied to the Lopital rule. In this regard, the equation for the total enthalpy on the axis of the jet takes the form

$$
\left.\frac{\partial H}{\partial \xi}\right|_{\psi=0}=\left.\frac{2}{\operatorname{Pr}} \rho v_{t} \frac{\partial^{2}}{\partial \psi^{2}}\left(H-f u^{2}\right)\right|_{\psi=0}
$$

We apply the implicit four-point approximation scheme and take into account the symmetry conditions

$$
\begin{gathered}
H_{i, 1}^{s}=H_{i,-1}^{s} \text { and } u_{i, 1}^{s-1}=u_{i,-1}^{s-1}: \\
\frac{H_{i, 0}^{s}-H_{i-1,0}}{h_{\xi}}=\frac{2 \rho_{i, 0}^{s-1} v_{t i}^{s-1}}{\operatorname{Pr} h_{\psi}^{2}}\left[H_{i, 1}^{s}-2 H_{i, 0}^{s}+H_{i,-1}^{s}-\right. \\
\left.=f\left(\left(u^{2}\right)_{i, 1}^{s-1}-2\left(u^{2}\right)_{i, 0}^{s-1}+\left(u^{2}\right)_{i,-1}^{s-1}\right)\right)= \\
=\frac{4 \rho_{i, 0}^{s-1} v_{t i}^{s-1}}{\operatorname{Pr} h_{\psi}^{2}}\left[H_{i, 1}^{s}-H_{i, 0}^{s}-f\left(\left(u^{2}\right)_{i, 1}^{s-1}-\left(u^{2}\right)_{i, 0}^{s-1}\right)\right) .
\end{gathered}
$$

We introduce the notation $\sigma=h_{\xi} / h_{\psi}^{2}$ and collect these members 


$$
\begin{aligned}
& H_{i, 0}^{s}\left(1+\frac{4 \sigma}{\operatorname{Pr}} \rho_{i, 0}^{s-1} v_{t i}^{s-1}\right)=\frac{4 \sigma}{\operatorname{Pr}} \rho_{i, 0}^{s-1} v_{t i}^{s-1} H_{i, 1}^{s}+H_{i-1,0}- \\
& -\frac{4 \sigma}{\operatorname{Pr}} \rho_{i, 0}^{s-1} v_{t i}^{s-1} f\left(\left(u^{2}\right)_{i, 1}^{s-1}-\left(u^{2}\right)_{i, 0}^{s-1}\right) .
\end{aligned}
$$

From here, we compose the first coefficients of direct sweep:

$$
\alpha_{0}^{(H)}=\frac{\frac{4 \sigma}{\operatorname{Pr}} \rho_{i, 0}^{s-1} v_{t}^{s-1}}{1+\frac{4 \sigma}{\operatorname{Pr}} \rho_{i, 0}^{s-1} v_{t}^{s-1}}, \beta_{0}^{(H)}=\frac{H_{i-1,0}-\frac{4 \sigma}{\operatorname{Pr}} \rho_{i, 0}^{s-1} v_{t i}^{s-1} f\left(\left(u^{2}\right)_{i, 1}^{s-1}-\left(u^{2}\right)_{i, 0}^{s-1}\right)}{1+\frac{4 \sigma}{\operatorname{Pr}} \rho_{i, 0}^{s-1} v_{t i}^{s-1}} .
$$

To approximate the equation in the internal nodes of the calculation, to reduce the recording volume, we introduce the notation $K=\frac{\rho^{2} v_{t} u}{\psi} y^{2}$. The square of the y coordinate is calculated as follows: $y_{j}^{2}=h_{\psi}^{2} \sum_{k=1}^{j}\left(\frac{k}{\rho_{i, k}^{s-1} u_{i, k}^{s-1}}+\frac{k-1}{\rho_{i, k-1}^{s-1} u_{i, k-1}^{s-1}}\right)$.

In general, we have

$$
K_{j}=\frac{\left(\rho_{i, j}^{s-1}\right)^{2} v_{t i}^{s-1} u_{i, j}^{s-1} h_{\psi}}{j} \sum_{k=1}^{j}\left(\frac{k}{\rho_{i, k}^{s-1} u_{i, k}^{s-1}}+\frac{k-1}{\rho_{i, k-1}^{s-1} u_{i, k-1}^{s-1}}\right)
$$

At $j=0$ values $y_{j}^{2}$ and $K_{j}$ taken equal to zero.

At $j=1$ we have $y_{1}=h_{\psi} / \sqrt{\rho_{i, 1}^{s-1} u_{i, 1}^{s-1}}$ and

$$
K_{1}=\frac{\left(\rho_{i, 1}^{s-1}\right)^{2} v_{t i}^{s-1} u_{i, 1}^{s-1} h_{\psi}}{1}\left(\frac{0}{\rho_{i, 0}^{s-1} u_{i, 0}^{s-1}}+\frac{1}{\rho_{i, 1}^{s-1} u_{i, 1}^{s-1}}\right)=\rho_{i, 1}^{s-1} v_{t i}^{s-1} h_{\psi}
$$

In connection with the introduction $K_{j}$ approximation of the equation in internal nodes with the second-order of accuracy in $\psi$ and first-order accuracy in $\xi$ has the form: 


$$
\begin{gathered}
\frac{H_{i, j}^{s}-H_{i-1, j}}{h_{\xi}}=\frac{1}{2 \operatorname{Pr} h_{\psi}^{2}}\left\{\left(K_{j}+K_{j+1}\right)\left[H_{i, j+1}^{s}-H_{i, j}^{s}-f\left(\left(u^{2}\right)_{i, j+1}^{s-1}-\left(u^{2}\right)_{i, j}^{s-1}\right)\right]-\right. \\
\left.-\left(K_{j}+K_{j-1}\right)\left[H_{i, j}^{s}-H_{i, j-1}^{s}-f\left(\left(u^{2}\right)_{i, j}^{s-1}-\left(u^{2}\right)_{i, j-1}^{s-1}\right)\right]\right\} .
\end{gathered}
$$

From here, we compose the finite difference equation

$$
\begin{gathered}
A_{j}^{(H)} H_{i, j+1}^{s}-B_{j}^{(H)} H_{i, j}^{s}+C_{j}^{(H)} H_{i, j-1}^{s}=D_{j}^{(H)}, \\
\text { where } A_{j}^{(H)}=\frac{\sigma}{2 \operatorname{Pr}}\left(K_{j}+K_{j+1}\right), C_{j}^{(H)}=\frac{\sigma}{2 \operatorname{Pr}}\left(K_{j-1}+K_{j}\right), \\
B_{j}^{(H)}=1+\frac{\sigma}{2 \operatorname{Pr}}\left(K_{j-1}+2 K_{j}+K_{j+1}\right), \\
D_{j}^{(H)}=-H_{i-1, j}+\frac{\sigma f}{2 \operatorname{Pr}}\left[\left(K_{j}+K_{j+1}\right)\left(\left(u^{2}\right)_{i, j+1}^{s-1}-\left(u^{2}\right)_{i, j}^{s-1}\right)-\left(K_{j}+K_{j-1}\right)\left(\left(u^{2}\right)_{i, j}^{s-1}-\left(u^{2}\right)_{i, j-1}^{s-1}\right)\right] .
\end{gathered}
$$

We believe that $H_{i, j-1}^{s}=\alpha_{j-1}^{(H)} H_{i, j}^{s}+\beta_{j-1}^{(H)}$.

Accordingly, we have

$$
A_{j}^{(H)} H_{i, j-1}^{s}-\left(B_{j}^{(H)}-C_{j}^{(H)} \alpha_{j-1}^{(H)}\right) H_{i, j}^{s}=D_{j}^{(H)}-C_{j}^{(H)} \beta_{j-1}^{(H)}
$$

From here, we find the values of the running coefficients

$$
\alpha_{j}^{(H)}=\frac{A_{j}^{(H)}}{B_{j}^{(H)}-C_{j}^{(H)} \alpha_{j-1}^{(H)}}, \quad \beta_{j}^{(H)}=\frac{-D_{j}^{(H)}+C_{j}^{(H)} \beta_{j-1}^{(H)}}{B_{j}^{(H)}-C_{j}^{(H)} \alpha_{j-1}^{(H)}} .
$$

According to these formulas, we perform calculations up to $j=N_{j}-1$. At $j=N_{j}$ the zero boundary condition is realized and then for $j=N_{j}-1 . .0$ reverse run

$$
H_{i, j}^{s}=\alpha_{j}^{(H)} H_{i, j+1}^{s}+\beta_{j}^{(H)}
$$

As you can see, the equations are approximated with the first order of accuracy in the longitudinal coordinate and the second order of accuracy in the radial coordinate. At the same time, conditions are provided that make it possible to apply the sweep method in the process of successive approximation.

The results obtained are in good agreement with experimental data on the distribution and combustion of a gas mixture in a satellite air stream. In this work, we demonstrated the option of controlling the combustion process by varying the gas mixture concentration in 
the combustible composition: with an increase in which the dimensions of the front and the temperature at the flame front increase, which corresponds to the physics of the process.

Accordingly, the presented method for calculating the velocity, total enthalpy, and concentration fields can be used in the study and control of a single and composite axisymmetric jet with the combustion of various combustible mixtures in the air or oxygenenriched air at various input data.

\section{Results and Discussion}

Calculations show that as fuel increases with gas temperature, the length of the flames decreases. Private $T_{1}=600 \mathrm{~K}$ When, $T_{2}=293.15 \mathrm{~K}$ and $T_{2}=400 \mathrm{~K}$ for their case $l^{*}=175.02$ and $l^{*}=135.05$ values were obtained. Conversely, increased air temperature leads to an increase in the length of the flame. The first is due to the reduction in fuel gas consumption, and the second is due to the high oxygen content of oxygen in the air. In general, the results show that the propane fuel combustion in the airflow has a significant difference between the temperature and the indicator area compared to other gases.

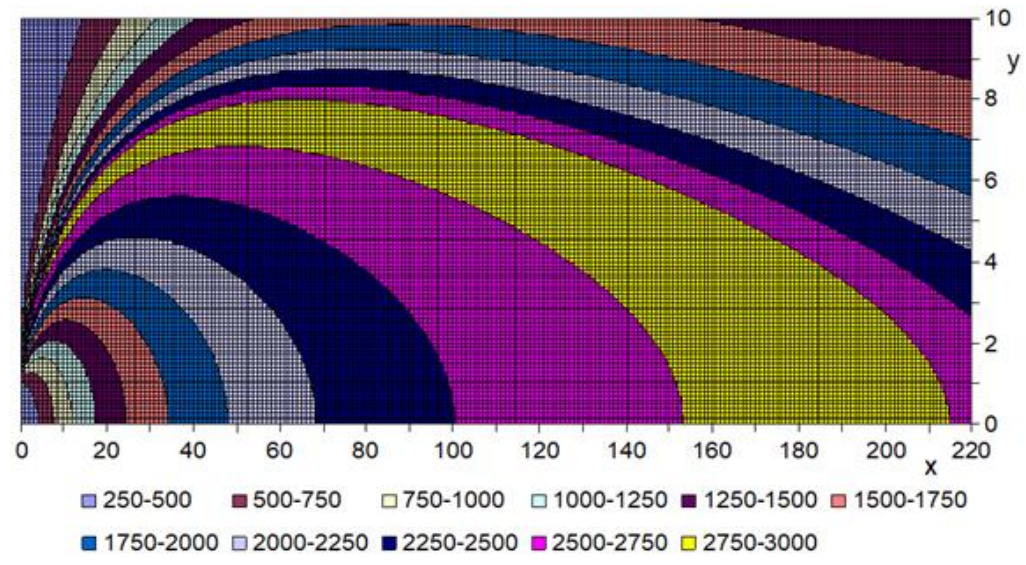

Fig. 1. $T_{1}=600 \mathrm{~K}$ and $T_{2}=293.15 \mathrm{~K}$ temperature distribution graph 


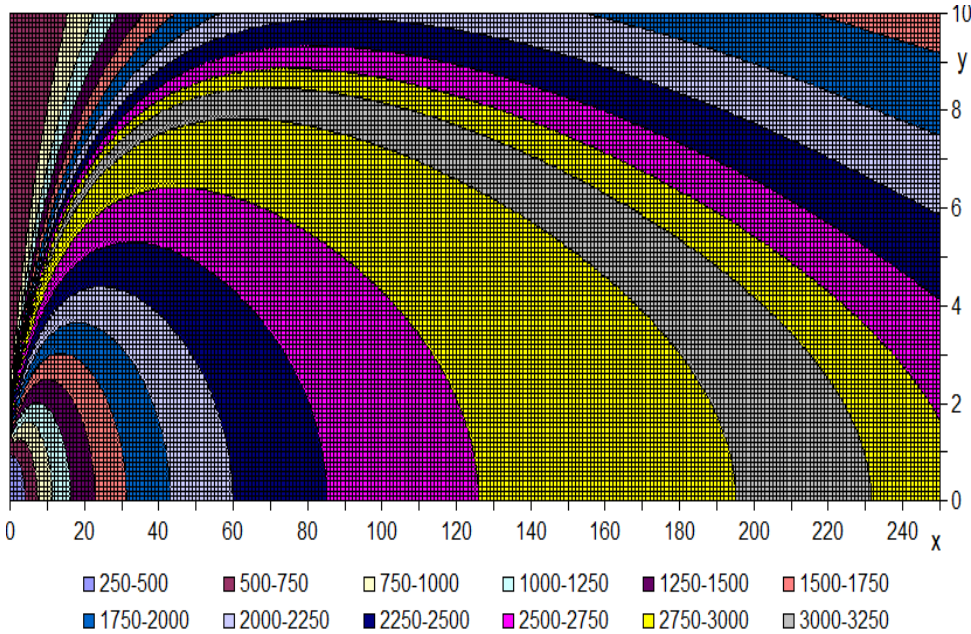

Fig. 2. $T_{1}=600 \mathrm{~K}$ and $T_{2}=400 \mathrm{~K}$ temperature distribution graph

In the article, we limit ourselves to discussing the effect of the temperature of the particular gases on isotherms and concentrations.

The starting values for the flow area corresponding to the concentration area and key components were determined. $T_{1}=600 \mathrm{~K}$ and $T_{2}=293.15 \mathrm{~K}$ while $\bar{x}=10, \bar{x}=40$ and $\bar{x}=200$ The graphs for the cross-sections are given. As you can see from this graph, $\bar{x}=10$ the fuel core is broken. The flame $\bar{r}=1.8$ expanded to. Next $\bar{x}=40$, as can be seen from the graph, there is a decrease in fuel over time, and the next $\bar{x}=200$ concentrations after the flame are given below.

\section{Conclusions}

The results obtained are in good agreement with experimental data on the distribution and combustion of a gas mixture in a satellite air stream. In this work, we demonstrated the option of controlling the combustion process by varying the gas mixture concentration in the combustible composition: with an increase in which the dimensions of the front and the temperature at the flame front increase, which corresponds to the physics of the process.

Accordingly, the presented method for calculating the velocity, total enthalpy, and concentration fields can be used in the study and control of a single and composite axisymmetric jet with the combustion of various combustible mixtures in the air or oxygenenriched air at various input data.

\section{References}

1. Vulis L.A., Ershin Sh.A., Yarin L.P. Fundamentals of the theory of a gas torch. - L: Energy, p 203 . (1968)

2. Pope S.B., Ten questions Concerning the Large-Eddy Simulation of Turbulent Flows, New Journal of Physics, 6 (35).(2004), 
3. Khuzhayev I.K. The development of mathematical models of diffusion combustion and gas transportation through the pipeline: Diss. Dr. tech. sciences. - Tashkent, p 336. (2009)

4. Roach P. Computational hydromechanics. - M.: Mir, p 612. (1980).

5. Samarsky A.A., Vabishchevich P.N. Computational heat transfer. - M .: URSS editorial,. P 784. (2009).

6. Zverev V.G., Goldin V.D. The difference scheme for solving convection-diffusion problems, Computational technology, 7, (6). pp 24-37. (2002).

7. Zh. Rui, G. Han, H. Zhang, S. Wang,H. Pu, and K. Ling, "A new model to evaluate two leak points in a gas pipeline, ” Journal of Natural Gas Science and Engineering, 46, pp. 491-497, (2017).

8. Gudich I.G., Zhukov V.T., Manukovsky K.V., Novikova N.D., Rykov Yu.G., Feodoritova O.B. Numerical simulation of a high-speed combustion chamber using the OpenFOAM package, KIAM Preprints named after M.V. Keldish.. pp 10. - 32 . / doi: 10.209448 / prepr-2016-10. (2016).

9. Yakimov A.S. Mathematical modeling of thermal protection and some heat and mass transfer problems. - Tomsk: Publishing house of Tomsk University, p 214. (2015).

10. Khujhayev I.K., Khamdamov M.M. A numerical method for solving the problem of an axisymmetric turbulent jet of a propane-butane mixture during diffusion burning, VViPM, Tashkent, p 4. (2018).

11. Khuzhayev I.K., Bozorov O.Sh. Features of the diffusion torch of multicomponent gas in a turbulent flow, Uzbek Journal of Problems of Mechanics. - Tashkent,. (4) pp.1722. (1998).

12. Khojaev I.K, Hamdamov M.M. Numerical Results Of Diffusion Combustion In Turbulent Flow Of Reacting Gases, International Journal of Advanced Science and Technology, 29, (9). pp. 2060-2074. (2020),

13. Aliev F., Zhumaev Z.Sh. Jet currents of reacting gases. Tashkent: Fan,. p 132. (1987).

14. Khojaev I.K, Hamdamov M.M. Numerical Method for Calculating Axisymmetric Turbulent Jets of Reacting Gases During Diffusion Combustion. Jour of Adv Research in Dynamical and Control Systems, 07, (12), (2020).

15. M.Hamdamov, I. Khujaev, O. Bazarov, K. Isabaev. Axisymmetric turbulent methane jet propagation in a wake air flow under combustion at a finite velocity. IPICSE. 1030(2021) 012163. Doi: 10.1088/1757-899X/1030/1/012163. (2021). 